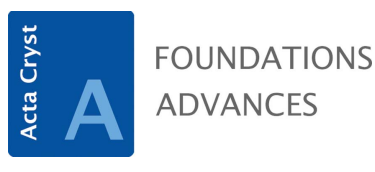

ISSN 2053-2733

Received 18 April 2016

Accepted 22 April 2016

Keywords: quotient graphs; reticular chemistry; crystal topology; materials design.

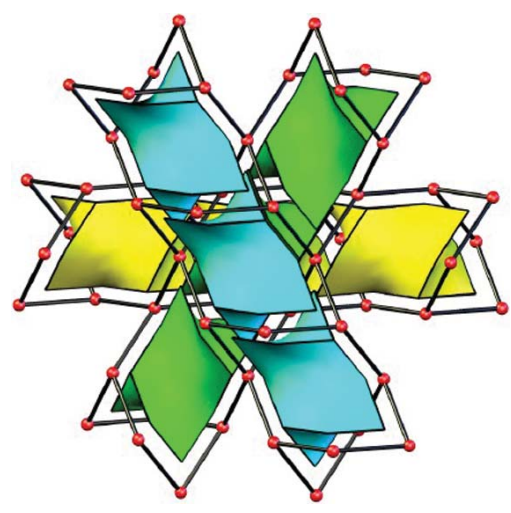

C 2016 International Union of Crystallography

\section{Simple graphs that guide combinatorial materials design}

\author{
Phillip M. Duxbury*
}

Department of Physics and Astronomy, Michigan State University, East Lansing, MI 48824, USA. *Correspondence e-mail: Duxbury@pa.msu.edu

Reticular synthesis (Yaghi et al., 2003) is producing a cornucopia of new crystalline materials due to the availability of a variety of structural units and linkers that can be combined in myriad ways to produce high-quality crystals. To harness the richness of this 'crystal engineering', simple representations of complex structures are needed to guide understanding and design (O'Keeffe \& Yaghi, 2012). In some cases topological features, such as minimum ring size, provide good guides to expected material properties, especially for nanoporous materials such as zeolites for catalytic applications. In this issue, building on a community effort dating back decades, Jean-Guillaume Eon (2016) reports on a finite 'quotient graph' representation of complex crystal structures, and demonstrates that this representation provides a significant reduction in graph complexity while preserving key topological properties such as minimum ring size.

Coordination polymers (Hagrman et al., 1999; Kitagawa et al., 2004) are combinations of structural units that can form bonds with three or more neighbors and linkers that are able to form two (and sometimes more) bonds. Metal centers are one example of the structural units, and when they are covalently bonded to organic linkers the resulting crystals are called metal-organic frameworks (MOFs) (Tranchemontagne et al., 2008), with a special subclass being zeolite-like structures linked by imidazolate ions (ZIFs). Combinations of covalent organic structural units with rigid organic linkers are called covalent organic frameworks (COFs). Reticular chemistry refers to a subclass of coordination polymers where the linkers are rigid and the crystal structures are robust, including MOFs, COFs and ZIFs; and they are of particular interest in the crystal engineering of new nanoporous catalysts and gas-storage materials (Yaghi et al., 2003).

The Reticular Chemistry Structure Resource (RCSR) (O'Keeffe et al., 2008) provides a database of crystal structures classified as nets, which are systematic reductions of the full crystal structure to a periodic graph. For example, the sodium chloride structure is represented as a simple cubic net where the identity of the sodium and chlorine ions is ignored so that each ion is represented by a vertex, and the specific length of the nearestneighbor bonds between $\mathrm{Na}$ and $\mathrm{Cl}$ is also ignored and replaced by a generic graph edge. Only the direct chemical bonds are represented by edges in the net. Further reductions include removing singly-connected edges and replacing a vertex connected to only two edges by a single edge. Graph theory depends only on connectivity, hence vizualization of nets in the RCSR corresponds to specific embeddings of the underlying nets, and where possible bonds of equal length are used in these embeddings. In all cases the embedding in RCSR is the maximum-symmetry embedding which leads to a specification of the coordinates and unit cell of the associated crystal structure with the simplest structure. At the time of writing, the RCSR database contains 2537 periodic three-dimensional (3D) nets (three-dimensional structures) and 83 periodic two-dimensional nets (twodimensional structures). Each entry in the database is assigned a three-letter descriptor, that may also also have a fourth letter added. The simple cubic net associated with the $\mathrm{NaCl}$ structure is assigned the descriptor pcu. Each entry in the database can be 'reticulated' by placing different units (such as polyhedra) on each vertex and chemical linkers on each edge, yielding a vast array of potential materials in the same net class. A second descriptor associated with each entry in the database is a list of four integers, pqrs (3D nets), where these integers refer, respectively, to the number of transitively (translationally) distinct vertices, edges, faces and tiles (3D nets only) in the embedding of the net. There are many possible tilings of a net. For example, the simple cubic net could be 

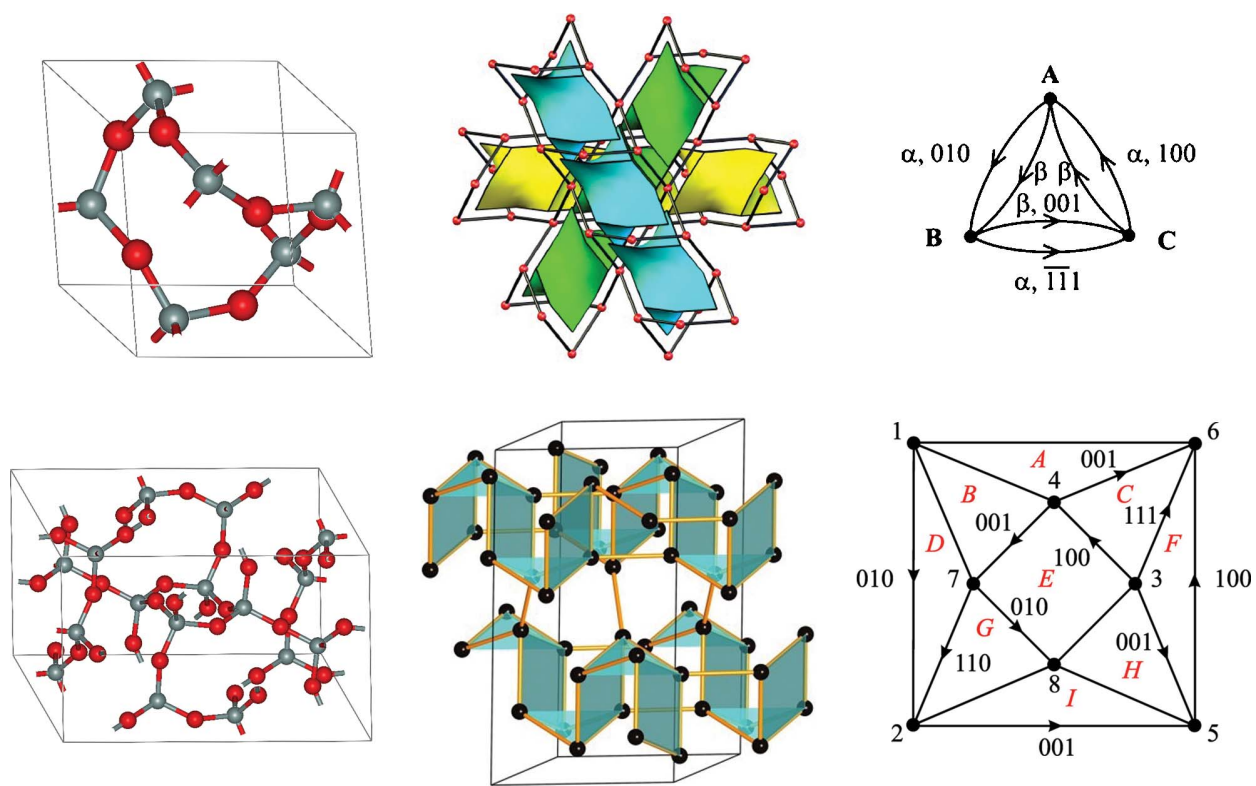

Figure 1

Different ways of representing the crystal structures of quartz (top row) and coesite (bottom row), which are crystal polymorphs of silica, $\mathrm{SiO}_{2}$. The lefthand figures are the usual atomic structures (grey is Si and red is O), the middle figures are representations given by the Reticular Chemistry Structure Resource (RCSR: qtz, coe), and the right-hand figures are quotient graphs. The quotient graph for quartz is reproduced from Eon (2012) with permission of Springer and the quotient graph for coesite is reproduced from Eon (2016).

tiled by a rectangular tile; however, it is useful to define the tile that leads to minimum transitivity and this tiling is called the natural tiling, which is conjectured to be unique. Transitivity indicates the complexity of the crystal structure with structures having minimum transitivity [1111], such as pcu, being the simplest. Nets that can be tiled (embedded) by regular polygons with transitivity [111] (2D) and [1111] (3D) are called regular nets, while those with transitivity [112] (2D) and [1112] (3D) are called quasi-regular nets. The only three regular tilings of the plane are by triangles, squares and polygons, while the only regular tiling of three-dimensional Euclidean space is by cubes.

The representation of crystal structures by nets provides a useful framework for thinking about the design of new materials. The net representation can also be used for more quantitative characterization of proposed new materials through calculation of embeddings and topological properties. Several software packages are available for this purpose, including GavrogSystre (http://www.gavrog.org) and ToposPro (Blatov et al., 2014). Since topological properties only depend on connectivity, the concept of distance in a graph plays a key role. In graph theory the distance between two vertices is the minimum number of edges that must be crossed in order to go from one vertex to the other, which is called the minimum path between the two vertices. A closely related concept is that of a simple cycle, which is the minimal number of edges that must be crossed, without repeated vertices or edges, in order to return to the same vertex. A circuit is similar, except that repeated vertices are allowed. In the chemistry and physics communities the term 'ring' is frequently used when referring to analogous objects, which can lead to some confusion as the term 'ring' in mathematics has a different meaning.
The quotient-graph representation of a net described in the article by Eon is a minimal representation, yet it contains full information about the net. Fig. 1 illustrates different ways of representing two crystal polymorphs of $\mathrm{SiO}_{2}$ $(\alpha$-quartz and coesite). The left-hand two images are the traditional atomic structures, the middle two figures are the networks with a minimum tiling from RCSR and the righthand two figures are the quotient graphs. The quotient graph is found by identifying the set of unique vertices in the net, and then identifying the unique edges that connect them. In order that the planar graphs in the right-hand figures can be used to reconstruct a 3D net, 'voltages' are associated with each edge. The voltage of the edge indicates the direction of the edge in three-dimensional Euclidean space. In the middle and righthand figures the oxygen linkers are replaced by an edge between the silicon atoms at their end points. The quotient graph clearly gives a neat summary of several key features of the crystal structure, for example the number of unique vertices (edges) in the nets is 3(6) for $\alpha$-quartz and 8(16) for coesite. Of more interest, however, is the fact that ring structure is also evident in the quotient graph, where for quartz there are three fundamental 6-rings, while in coesite there are four fundamental 4-rings and two fundamental 6-rings, up to translation. In more complex crystal structures, such as zeolites, identification of ring size is important and a quotient-graph analysis is clearly useful for this purpose. The compact form of quotient graphs leads to possible classification schemes based on them, as well as their use in the prediction of crystal structures with desirable ring designs, coordination sequences and other topological properties. The article by Eon provides an accessible and convincing introduction to this relatively under-utilized approach to combinatorial materials design. 


\section{scientific commentaries}

\section{References}

Blatov, V. A., Shevchenko, A. P. \& Proserpio, D. M. (2014). Cryst. Growth Des. 14, 3576-3586.

Eon, J.-G. (2012). Struct. Chem. 23, 987-996.

Eon, J.-G. (2016). Acta Cryst. A72, 268-293.

Hagrman, P. J., Hagrman, D. \& Zubieta, J. (1999). Angew. Chem. Int. Ed. 38, 2638-2684.
Kitagawa, S., Kitaura, R. \& Noro, S. (2004). Angew. Chem. Int. Ed. 43, 2334-2375.

O'Keeffe, M., Peskov, M. A., Ramsden, S. J. \& Yaghi, O. M. (2008). Acc. Chem. Res. 41, 1782-1789.

O'Keeffe, M. \& Yaghi, O. M. (2012). Chem. Rev. 112, 675-702.

Tranchemontagne, D. J., Ni, Z., O'Keeffe, M. \& Yaghi, O. M. (2008). Angew. Chem. Int. Ed. 47, 5136-5147.

Yaghi, O. M., O'Keeffe, M., Ockwig, N. W., Chae, H. K., Eddaoudi, M. \& Kim, J. (2003). Nature, 423, 705-714. 